\section{Assessment of an in vitro physiological relevant model to check therapeutic strategies for glaucoma}

\author{
Sara Tirendi,,1,2 Stefania Vernazza, 1,3 \\ Sergio Saccà, 4 Anna Maria Bassi ${ }^{1,2}$ \\ 1Department of Experimental Medicine \\ (DIMES), University of Genoa, Genoa; \\ 2Inter-University Center for the \\ Promotion of the 3Rs Principles in \\ Teaching and Research (Centro 3R); \\ 3IRCCS, Fondazione G.B. Bietti, Rome; \\ ${ }^{4}$ Ophthalmology Unit, Polyclinic San \\ Martino Hospital, Genoa, Italy
}

\begin{abstract}
Glaucoma is a chronic, progressive and heterogeneous optic neuropathy which affects in the early stage the peripheral vision and then the central vision, leading to irreversible blindness. As known, in glaucoma the trabecular meshwork represent the main tissue which is impaired by chronic oxidative stress, aging and increase of intraocular pressure. Today, the lack of human-based models, with characteristics of high repeatability and reproducibility as well, called for an high-quality in vitro model with a good degree of resemblance for the tissue or organ of interest as a basis for new drug testing. Our team has been committed to this purpose by assessment of an in vitro $3 \mathrm{D}$ TM human-based model, closer to in vivo, using millifluidic technology, to better identify the key events underlying the pathogenesis of glaucoma and to evaluate new therapies targeted at disease treatment and prevention.
\end{abstract}

\section{Introduction}

Glaucoma is the second cause of blindness in the world affecting over 67 million people worldwide. 1 As known, the main causes of glaucoma onset are oxidative stress and vascular alteration which impaired Trabecular meshwork activities.
The oxidative damage is an important step in pathogenesis of Primary Open Angle Glaucoma and might be a relevant target for both prevention and therapy. ${ }^{2}$

Therefore, the aim of this study was to develop an in vitro $3 \mathrm{D}$ human-based dynamic model of trabecular meshwork to define the key elements relating to the glaucoma onset.

\section{Materials and Methods}

3D cultures of Human Trabecular Meshwork Cells (HTMC, Cell APPLICATION INC). Were made by embedding HTMCs into $100 \%$ Corning Matrigel ${ }^{\mathrm{TM}}$ Matrix and were maintained in a millifluidic bioreactor system connected to the peristaltic pump (Live Box 1 and Live Flow, IV-Tech srl) with constant flow rate. ${ }^{3}$ To simulate chronic stress conditions 3DHTMCcultures were exposed to $\mathrm{H}_{2} \mathrm{O}_{2}$ treatment $(500 \mu \mathrm{M})$ for 2 hours followed by 22 hours of recovery, until 15 days.

\section{Results}

Confocal imaging analysis and Alamar blue assay, ${ }^{4}$ as index of proliferation/metabolic state of cultures, evidenced a good healthy state of HTMCs. Moreover, in our dynamic model an efficient response to stress was shown, since it was observed a NF-kB and TNF- $\alpha$ activation. To evaluate the feasibility of our dynamic HTMC 3Dmodel as a useful tool for evaluate therapeutic strategies for glaucoma disease, we analyzed the effects of a polyphenol mixture (PM), an active compound of a commercial eye drops for glaucoma. For this purpose, we studied the biological property of PM in counteracting chronic oxidative stress on 3D HTMCs. Preliminary qPCR analysis showed a modulation of gene levels of collagens and other ECM glycoproteins.

Taking into account these findings, our dynamic 3D-HTMC model can provide useful information on new prevention and therapeutic strategies for glaucoma.
Correspondence: Sara Tirendi, Department of Experimental Medicine (DIMES), University of Genoa, Genoa, Italy.

E-mail: tirendisara@gmail.com

Key words: Glaucoma; in vitro model; oxidative stress; toxicology.

Conference presentation: this paper was presented at the Second Centro 3R Annual Meeting - 3Rs in Italian Universities, 2019, June 20-21, University of Genoa, Italy.

Received for publication: 28 October 2019. Accepted for publication: 11 November 2019.

This work is licensed under a Creative Commons Attribution NonCommercial 4.0 License (CC BY-NC 4.0).

C Copyright: the Author(s), 2019

Licensee PAGEPress, Italy

Biomedical Science and Engineering 2019; 3(s3):120 doi:10.4081/bse.2019.120

\section{References}

1. Awai-Kasaoka N, Inoue $\mathrm{T}$, Kameda $\mathrm{T}$, et al. Oxidative stress response signaling pathways in trabecular meshwork cells and their effects on cell viability. Mol Vis 2013;19:1332-40.

2. Keller KE, Bhattacharya SK, Borrás T, et al. Consensus recommendations for trabecular meshwork cell isolation, characterization and culture. Exp Eye Res 2018;171:164-73.

3. Ucciferri U, Sbrana T, Ahluwalia A. Allometric Scaling and Cell Ratios in Multi-Organ in vitro Models of Human Metabolism. Front Bioeng Biotechnol 2014;2:74.

4. Bonnier F, Keating ME, Wróbel TP, et al. Cell viability assessment using the Alamar blue assay: a comparison of 2D and 3D cell culture models. Toxicol Vitro Int $\mathrm{J}$ Publ Assoc BIBRA 2015;29:124-31. 